\title{
Supplemental Information: Perturbation of ACE2 structural ensembles by SARS-CoV-2 spike protein binding
}

\author{
Arzu Uyar, Alex Dickson
}

April 2, 2021 
Table S1: Isomerization states of PRO145 of ACE2 for solved structure in the literature. Isomerization states were determined using the corresponding $\omega$ backbone angle, with a cutoff of 0.5 radians from the basins defining cis $(0.0)$ and trans $( \pm \pi)$. Angles outside of this range are denoted "int" for intermediate. Note that trimer oligomerization states are engineered trimers of ACE2 protease domains [1].

\begin{tabular}{|c|c|c|c|c|}
\hline PDB ID & Iso. state & Olig. state & Spike bound & Method \\
\hline $1 \mathrm{r} 42$ & cis & monomer & apo & X-ray \\
\hline $1 \mathrm{r} 4 \mathrm{l}$ & cis & monomer & apo & X-ray \\
\hline 2ajf & cis & monomer & apo & X-ray \\
\hline $3 \mathrm{~d} 0 \mathrm{~g}$ & cis & monomer & SARS-CoV & X-ray \\
\hline $3 \mathrm{~d} 0 \mathrm{~h}$ & cis & monomer & SARS-CoV & X-ray \\
\hline $3 \mathrm{~d} 0 \mathrm{i}$ & cis & monomer & SARS-CoV & X-ray \\
\hline $3 \mathrm{kbh}$ & int & monomer & NL63 & X-ray \\
\hline $3 \mathrm{sci}$ & cis & monomer & SARS-CoV & X-ray \\
\hline $3 \mathrm{scj}$ & cis & monomer & SARS-CoV & X-ray \\
\hline $3 \mathrm{sck}$ & cis & monomer & SARS-CoV & X-ray \\
\hline $3 \mathrm{scl}$ & cis & monomer & SARS-CoV & X-ray \\
\hline 6 acg & cis & monomer & SARS-CoV & Cryo-EM \\
\hline $6 a c j$ & cis & monomer & SARS-CoV & Cryo-EM \\
\hline 6ack & cis & monomer & SARS-CoV & Cryo-EM \\
\hline $6 \operatorname{cs} 2$ & cis & monomer & SARS-CoV & Cryo-EM \\
\hline $6 \mathrm{lzg}$ & cis & monomer & SARS-CoV-2 & X-ray \\
\hline $6 \mathrm{~m} 0 \mathrm{j}$ & cis & monomer & SARS-CoV-2 & X-ray \\
\hline $6 \mathrm{~m} 17$ & trans & dimer & SARS-CoV-2 & Cryo-EM \\
\hline $6 \mathrm{~m} 18$ & int & dimer & apo & Cryo-EM \\
\hline $6 \mathrm{~m} 1 \mathrm{~d}$ & $\operatorname{trans}$ & dimer & SARS-CoV-2 & Cryo-EM \\
\hline 6vw1 & cis & monomer & SARS-CoV-2 & X-ray \\
\hline $7 a 91$ & cis & monomer & SARS-CoV-2 & Cryo-EM \\
\hline $7 a 92$ & cis & monomer & SARS-CoV-2 & Cryo-EM \\
\hline $7 \mathrm{a} 94$ & cis & monomer & SARS-CoV-2 & Cryo-EM \\
\hline $7 a 95$ & cis & monomer & SARS-CoV-2 & Cryo-EM \\
\hline $7 a 96$ & cis & monomer & SARS-CoV-2 & Cryo-EM \\
\hline $7 \mathrm{a} 97$ & cis & monomer & SARS-CoV-2 & Cryo-EM \\
\hline $7 a 98$ & cis & monomer & SARS-CoV-2 & Cryo-EM \\
\hline $7 \mathrm{c} 8 \mathrm{~d}$ & cis & monomer & SARS-CoV-2 & Cryo-EM \\
\hline $7 \mathrm{ct} 5$ & trans & trimer & SARS-CoV-2 & Cryo-EM \\
\hline $7 \mathrm{kj} 2$ & trans & trimer & SARS-CoV-2 & Cryo-EM \\
\hline $7 \mathrm{kj} 3$ & trans & trimer & SARS-CoV-2 & Cryo-EM \\
\hline $7 \mathrm{kj} 4$ & trans & trimer & SARS-CoV-2 & Cryo-EM \\
\hline $7 \mathrm{kmb}$ & trans & monomer & SARS-CoV-2 & Cryo-EM \\
\hline $7 \mathrm{kms}$ & trans & monomer & SARS-CoV-2 & Cryo-EM \\
\hline $7 \mathrm{kmz}$ & trans & monomer & SARS-CoV-2 & Cryo-EM \\
\hline $7 \mathrm{knb}$ & trans & monomer & SARS-CoV-2 & Cryo-EM \\
\hline
\end{tabular}


Table S2: Training-testing accuracy table for LDA method. "DR" denotes that the DESRES dataset was included in the training.

\begin{tabular}{l|r|r|ll|ll} 
& & & \multicolumn{2}{l}{ training accuracy } & \multicolumn{2}{l}{ testing accuracy } \\
\hline Predictor & $n_{\text {atoms }}$ & $n_{\text {train }}$ & apo & complex & apo & complex \\
\hline CA-592 & 592 & 5 & $1.000 \pm 0.000$ & $1.000 \pm 0.000$ & $0.990 \pm 0.008$ & $0.992 \pm 0.007$ \\
\hline CA-592 & 592 & 15 & 1.000 & 1.000 & - & - \\
\hline CA-590 & 590 & 5 & $1.000 \pm 0.000$ & $1.000 \pm 0.000$ & $0.966 \pm 0.024$ & $0.966 \pm 0.022$ \\
\hline CA-590 & 590 & 15 & 1.000 & 1.000 & - & - \\
\hline CA-592-DR & 592 & 5 & $1.000 \pm 0.000$ & $1.000 \pm 0.000$ & $0.9813 \pm 0.014$ & $0.976 \pm 0.024$ \\
\hline CA-592-DR & 592 & 15 & 1.000 & 1.000 & - & - \\
\hline CA-INT & 80 & 5 & $0.88 \pm 0.10$ & $0.93 \pm 0.02$ & $0.83 \pm 0.09$ & $0.74 \pm 0.20$ \\
\hline CA-INT & 80 & 15 & 0.85 & 0.89 & - & - \\
\hline CA/SC-INT & 158 & 5 & $0.986 \pm 0.013$ & $0.993 \pm 0.004$ & $0.908 \pm 0.048$ & $0.84 \pm 0.14$ \\
\hline CA/SC-INT & 158 & 15 & 0.968 & 0.982 & - & -
\end{tabular}


Table S3: Chemical structure of ACE2 ligands simulated in the D. E. Shaw Research dataset [2]. Note that ligands doxazosin and pimecrolimus each had two trajectories, starting from different binding sites.

\begin{tabular}{|c|c|c|c|c|c|}
\hline Name & ChEMBL ID & Structure & Av. LDA (CA-590) & Av. LDA (CA/SC-INT) & Binding site \\
\hline acalabrutinib & CHEMBL3707348 & & -0.14 & 1.01 & 3 \\
\hline aclidinium & CHEMBL1194325 & & 2.04 & 2.97 & 3 \\
\hline afeletecan & CHEMBL134380 & & 0.04 & 2.75 & 3 \\
\hline amentoflavone & CHEMBL63354 & & -4.42 & -1.60 & 5 \\
\hline azathioprine & CHEMBL1200400 & & 2.26 & -1.03 & 3 \\
\hline barusiban & CHEMBL2218898 & & -0.34 & 3.01 & 3 \\
\hline
\end{tabular}




S CHEMBL1237048



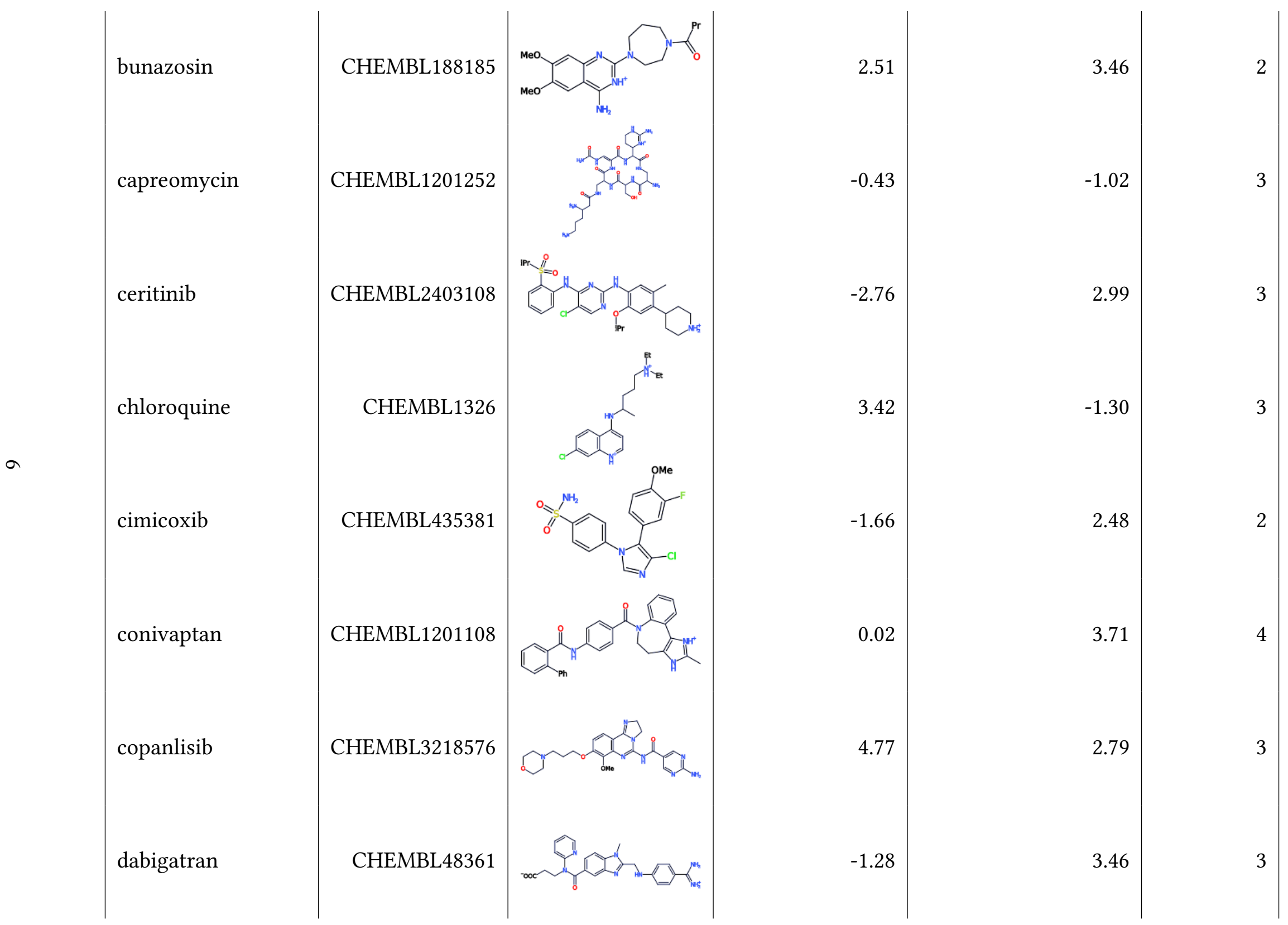


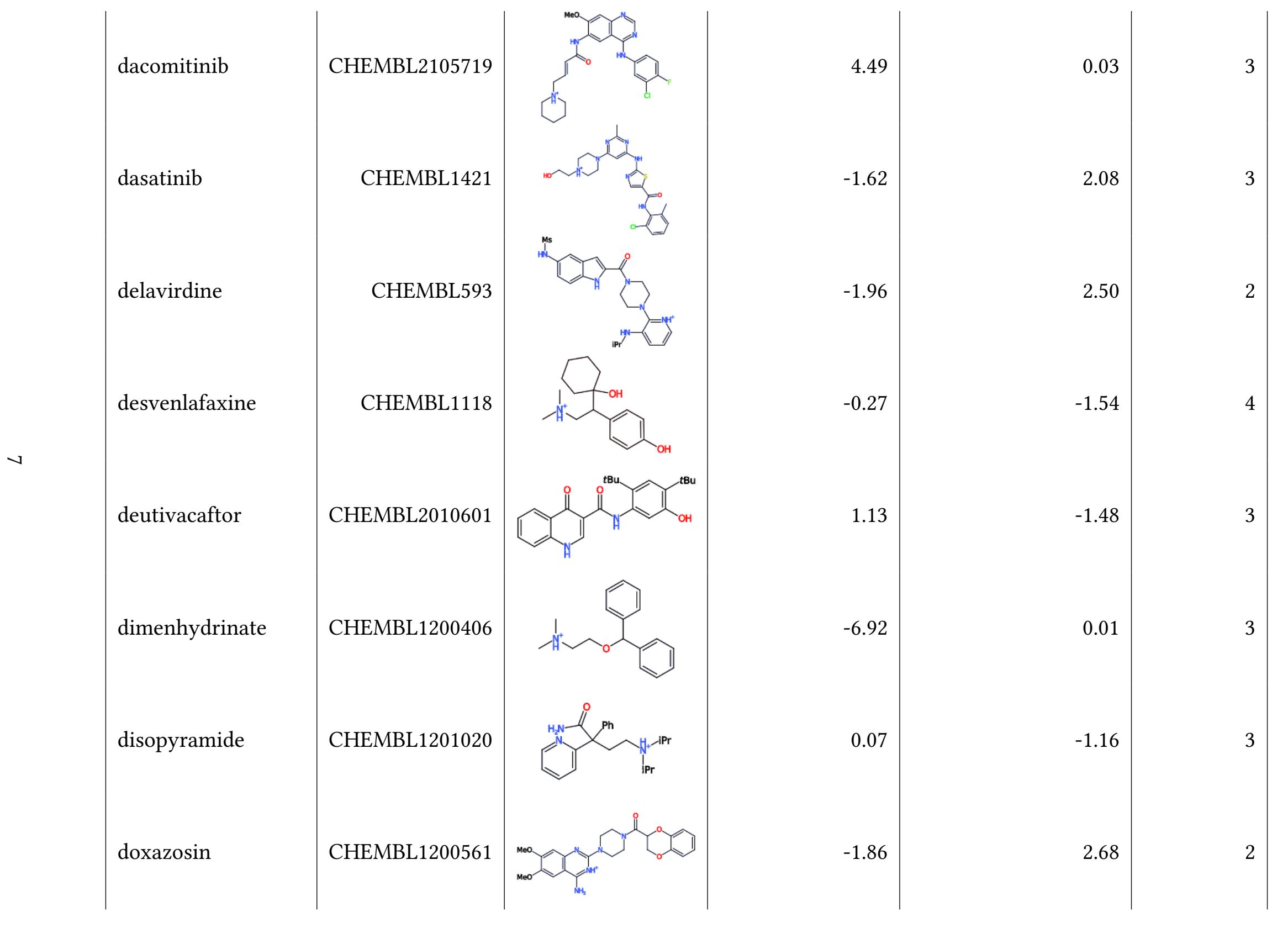




(CHEMBL1200561




(CHEMBL329592




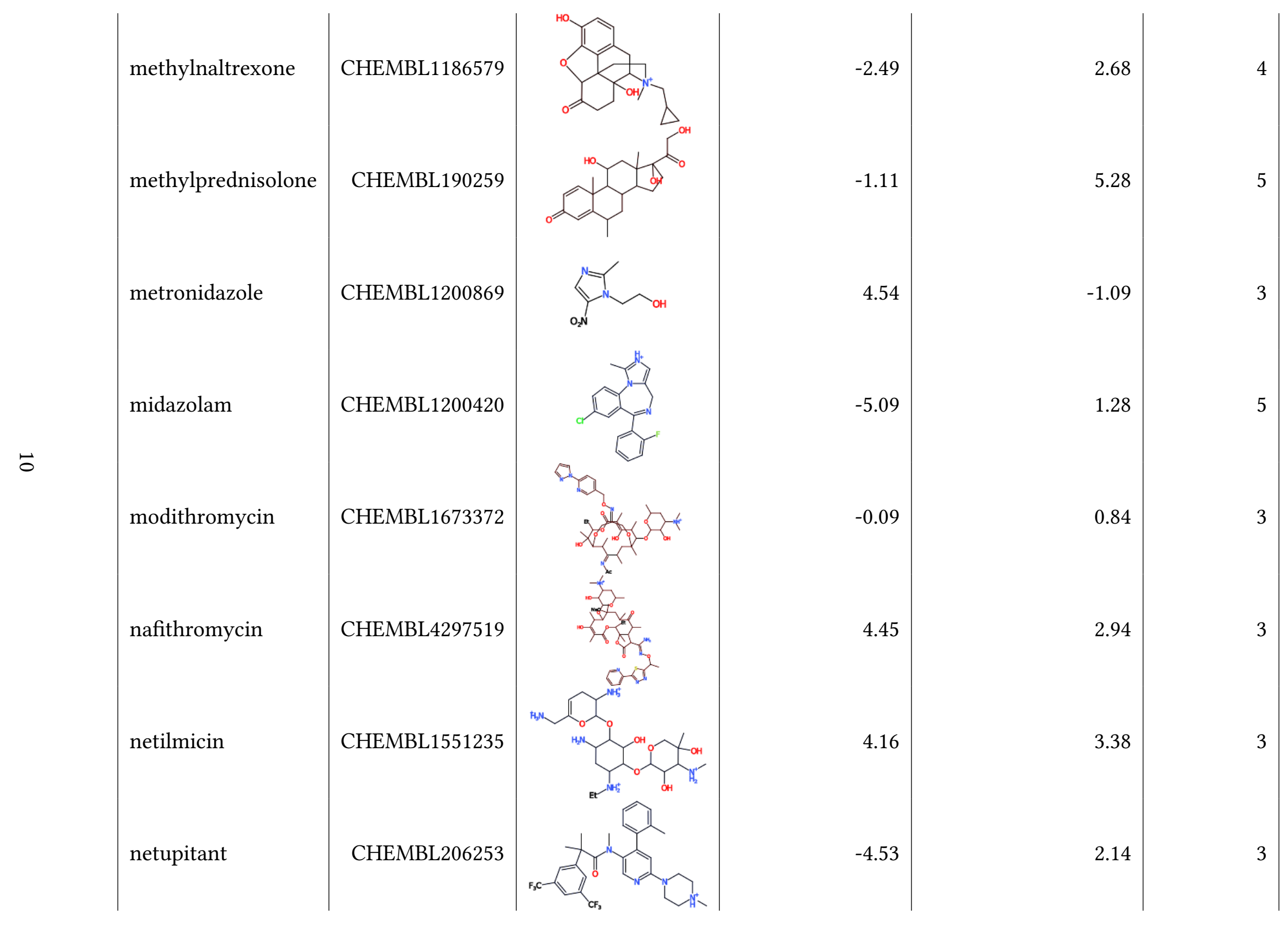



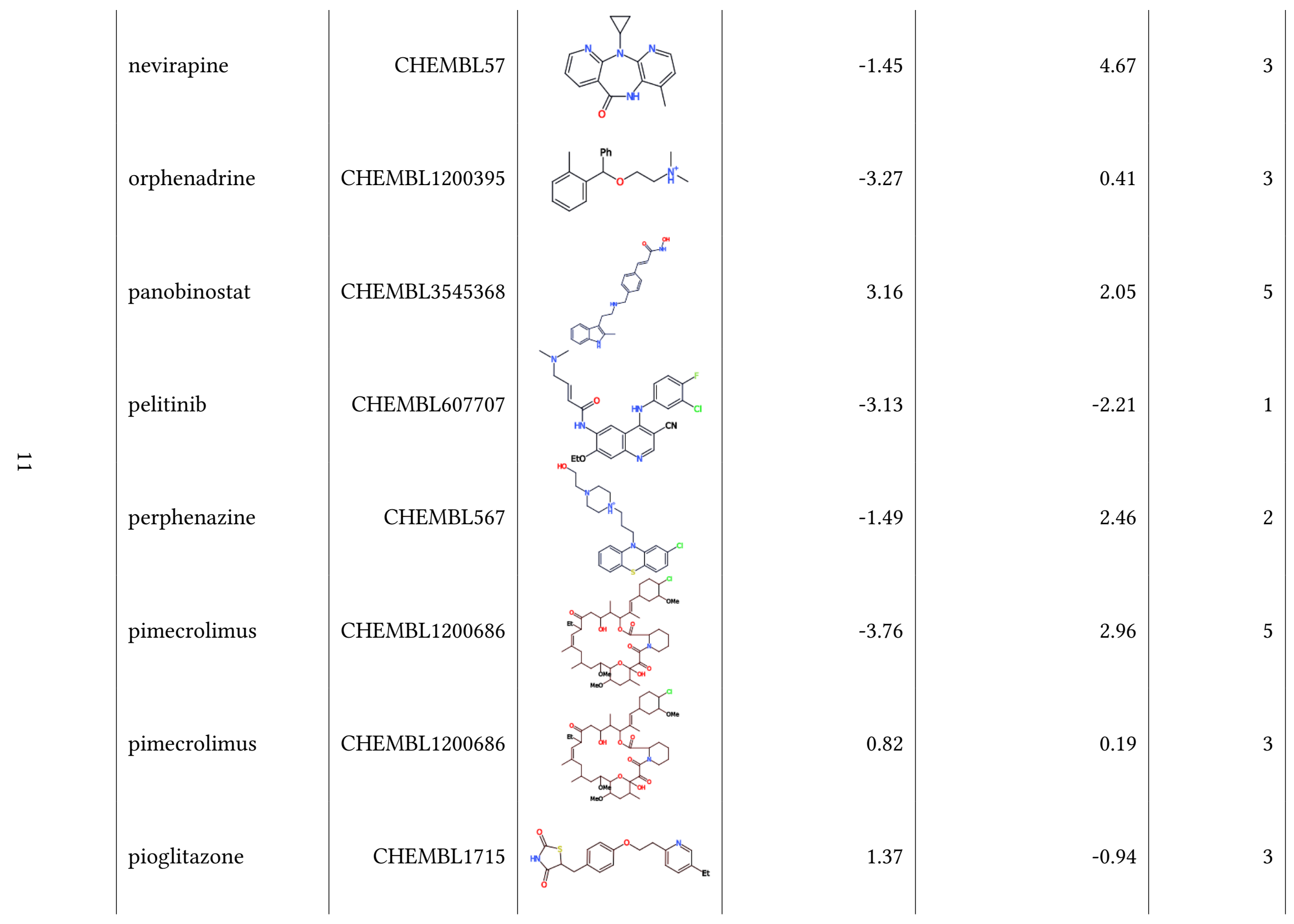

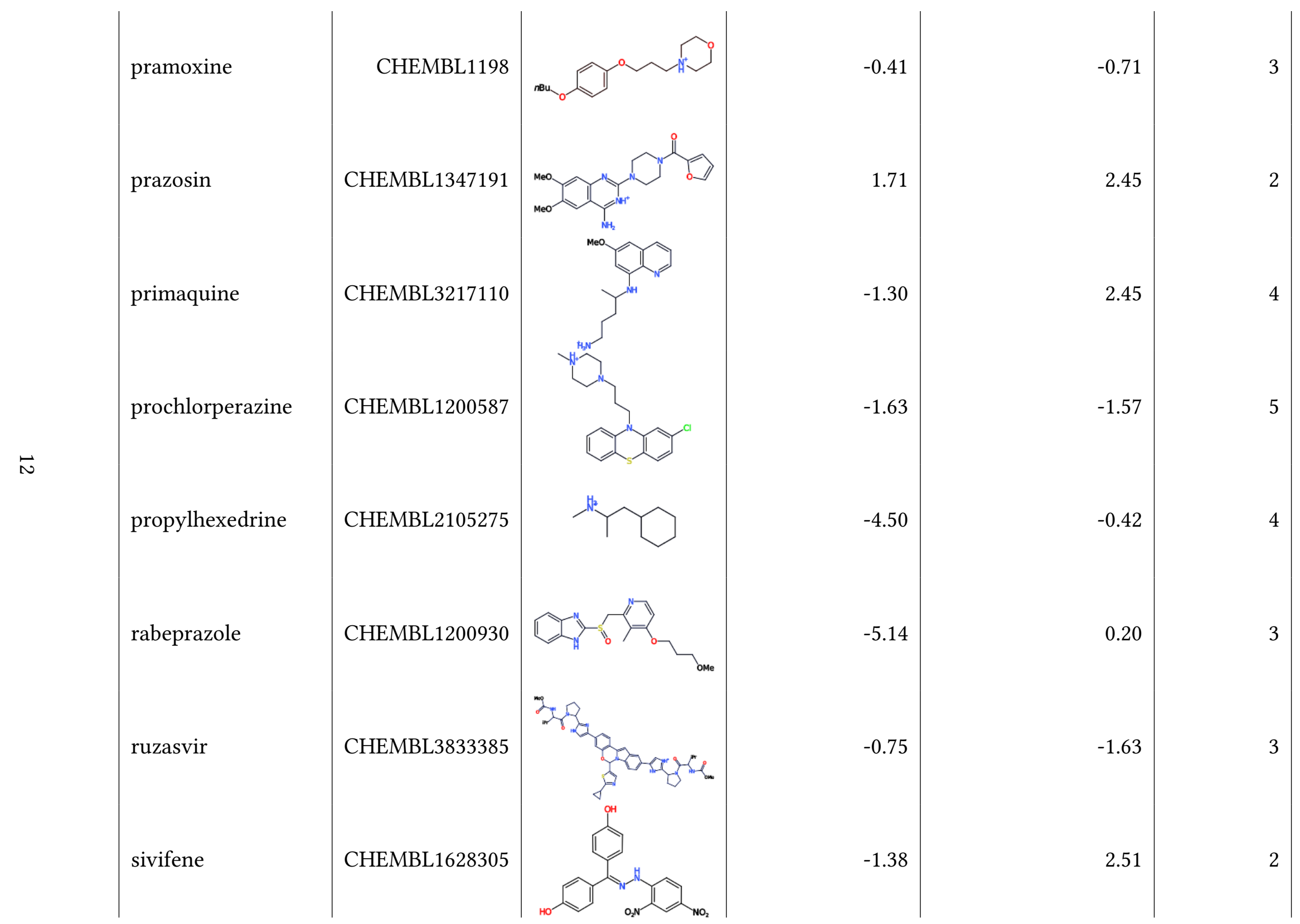

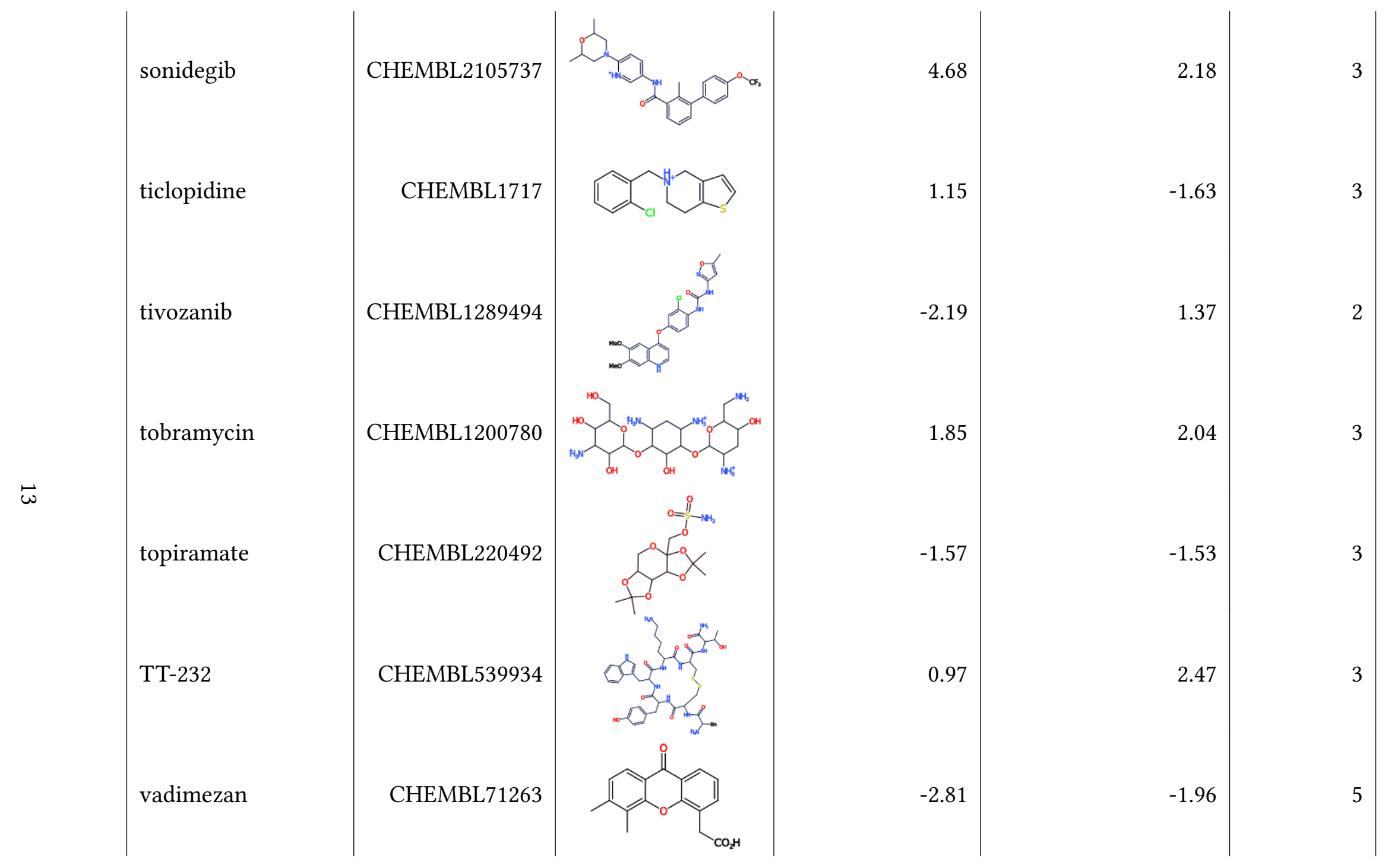

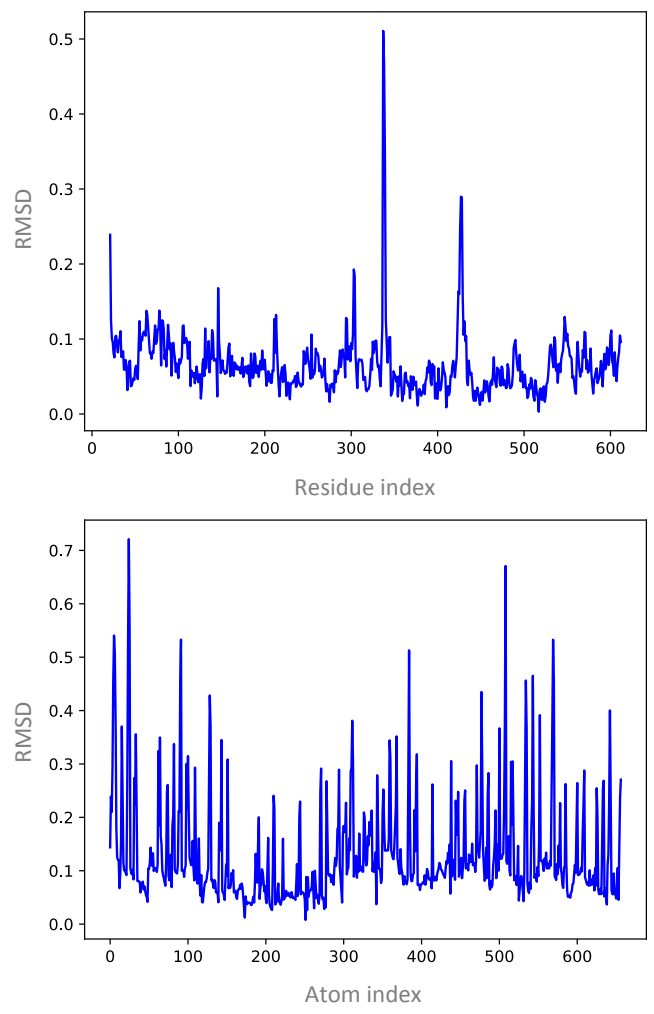

Figure S1: $\quad$ C $\alpha$ Residue-level RMSD profiles between the apo and the complex ACE2 structures (top). All-atom RMSD for atoms in $\mathrm{N}$-terminus helices (H1 and $\mathrm{H} 2$ : residues 21-100) (bottom). 

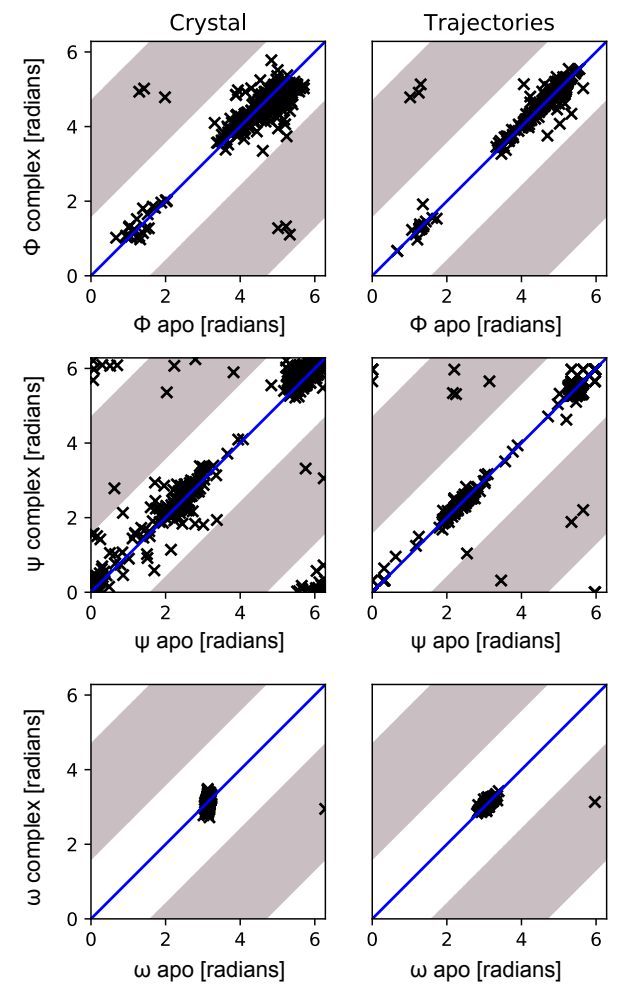

Figure S2: Backbone dihedral angle comparison between apo and complex forms of ACE2. Scatter plots compare the dihedral angles of ACE2 alone (apo) vs ACE2 bound to the S1 protein (complex). Differences obtained between the crystal structures are shown on the left and differences in MD trajectories are shown on the right. Areas with significant differences (greater than $\pm \pi / 2$ ) are shown in grey. Differences are examined separately for dihedral angles $\phi$ (top), $\psi$ (middle) and $\omega$ (bottom). 

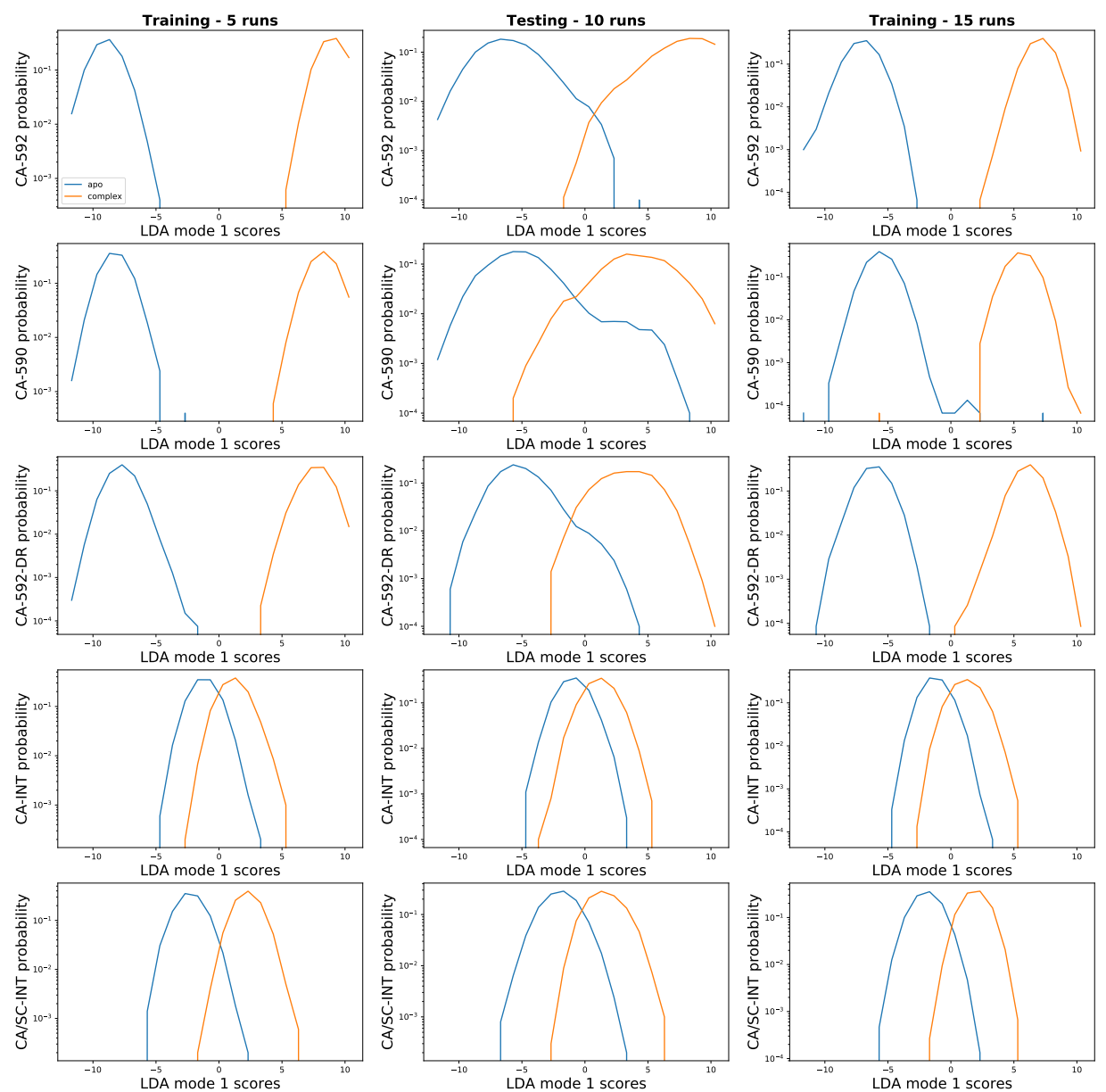

Figure S3: Probability distributions of LDA projections. Training sets are randomly chosen from three subsets of five runs. 


\section{References}

[1] Tianshu Xiao, Jianming Lu, Jun Zhang, Rebecca I. Johnson, Lindsay G.A. McKay, Nadia Storm, Christy L. Lavine, Hanqin Peng, Yongfei Cai, Sophia Rits-Volloch, Shen Lu, Brian D. Quinlan, Michael Farzan, Michael S. Seaman, Anthony Griffiths, and Bing Chen. A trimeric human angiotensin-converting enzyme 2 as an anti-sars-cov-2 agent in vitro, 2020.

[2] D. E. Shaw Research. Molecular Dynamics Simulations Related to SARS-CoV-2, 2020. 

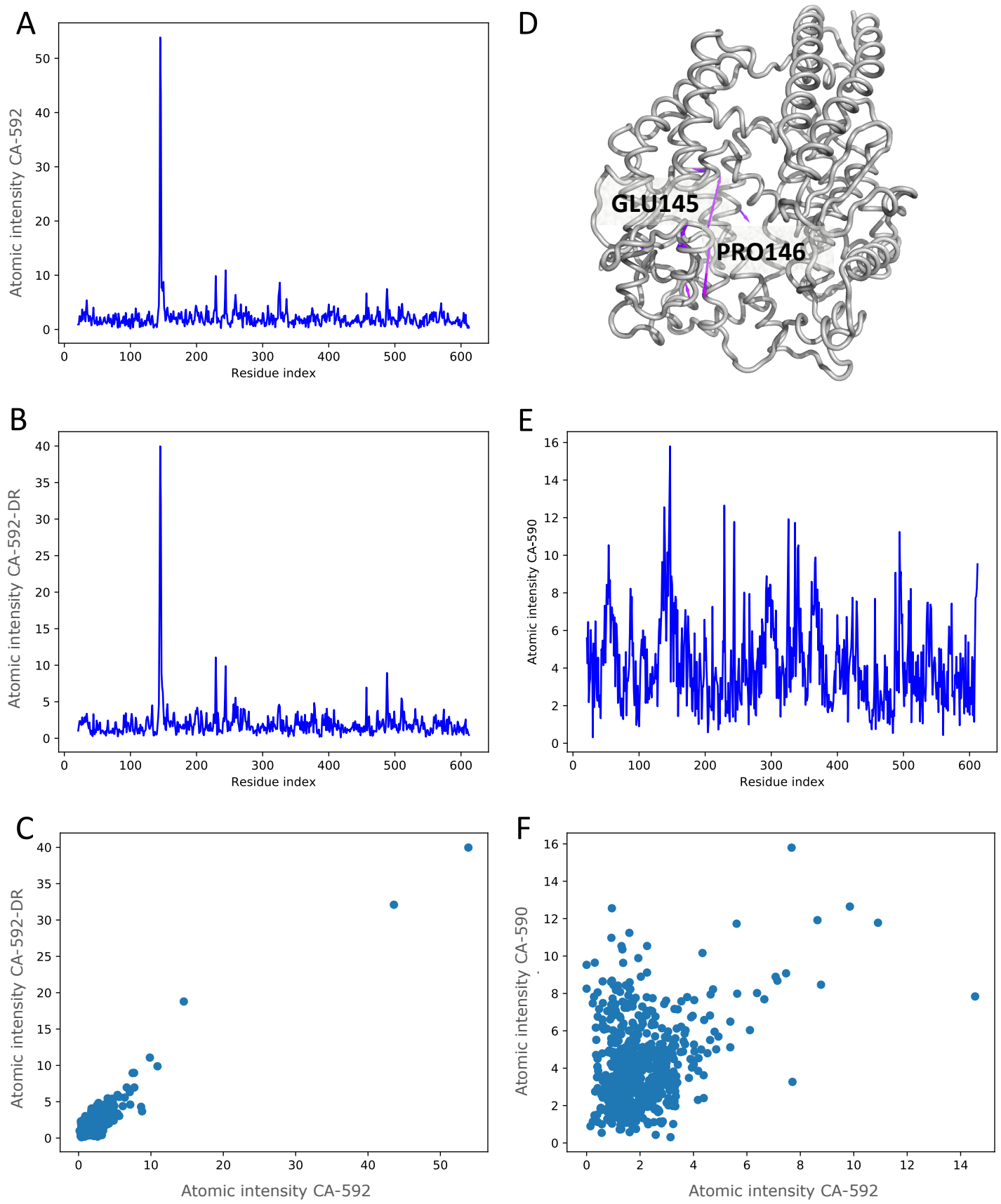

Figure S4: (A) Atomic intensity values of LDA CA-592. (B) Atomic intensity values of LDA CA-592-DR. (C) Comparison of atomic intensity values between CA-592 and CA592-DR. (D) Mode vector representation of LDA CA-592-DR. (E) Atomic intensity values of LDA CA-590. (F) Comparison of atomic intensity values between CA-592 and CA-590. 